\title{
Piston-effect-induced thermal jets in near-critical fluids
}

\author{
T. Fröhlich \\ European Aeronautic Defence and Space Company, 37 boulevard de Montmorency, 75016, Paris, France \\ D. Beysens* \\ ESEME, Service des Basses Températures, CEA Grenoble, Grenoble, France \\ and Laboratoire de Physique et Mécanique des Milieux Héterogènes, Ecole Supérieure de Physique et Chimie Industrielle, \\ 10, rue Vauquelin, 75231 Paris Cedex 5, France \\ Y. Garrabos \\ ESEME, Institut de Chimie de la Matière Condensée de Bordeaux, UPR 9048, CNRS, Université Bordeaux I, \\ 87, Avenue du Dr. Schweitzer, 33608 Pessac Cedex, France
}

(Received 26 April 2006; published 25 October 2006)

\begin{abstract}
In very compressible fluids, such as fluids near their critical point, the bulk fluid is adiabatically thermalized by the expansion of a hot boundary layer. Thanks to this thermomechanical process (the so-called piston effect) the fluid velocity at the edge of the boundary layer can become very high when the heating power is concentrated in a fissure. Spectacular jets are then observed in $\mathrm{SF}_{6}$ and $\mathrm{CO}_{2}$. Data obtained under weightlessness (in order to remove convection) and data obtained under earth gravity are compared and analyzed. They emphasize the key role of the boundary layer expansion for thermal phenomena in compressible fluids and the hydrodynamic nature of the piston effect.
\end{abstract}

DOI: 10.1103/PhysRevE.74.046307

PACS number(s): 47.40.-x, 05.70.Jk, 65.40.De, 64.70.Fx

\section{INTRODUCTION}

Near the liquid-gas critical point, fluids exhibit quite unusual properties. In particular, they behave as dilatable and compressible gases with liquidlike density. This is due to the critical divergence of a number of thermodynamic properties such as the isothermal compressibility and the isobaric thermal expansion coefficient.

Transport phenomena are also affected. The critical slowing down of the thermal transport is a well-known example. Because of the extremely low thermal diffusivity, a simple calculation shows that it would need more than one month to reach thermal equilibration in a critical (i.e., $\rho=\rho_{c}$ ) $\mathrm{CO}_{2}$ sample of $1 \mathrm{~cm}^{3}$ at $T-T_{c}=1 \mathrm{mK}$. ( $T$ is temperature and $T_{c}$ is the critical temperature; $\rho$ is density and $\rho_{c}$ is the critical density.) At the beginning of the space experiments under weightlessness, it thus seemed hopeless for scientists to try to homogenize, in temperature and density, a sample of fluid close to its critical point in a reasonable experimental time. However, Onuki et al. [1] pointed out the thermodynamic importance of the adiabatic heating of a closed cell. Close to the sample wall, heat diffusion makes a thin hot boundary layer (HBL) expand and compress adiabatically the rest of the fluid. A spatially uniform heating of the bulk fluid was indeed observed [2]. Simultaneously, a more detailed hydrodynamic mechanism of thermalization, which should proceed at the velocity of sound, was proposed by Zappoli et al. [3]. There should be a real flow at the border of the expanding diffusive layer and compressed bulk fluid. Other reports $[4,5]$ appeared nearly at the same time. This thermomechanical heating phenomenon was called the piston effect [3] as

\footnotetext{
*Corresponding author. Email address: daniel.beysens@espci.fr
}

the HBL acts as a piston. These developments allowed the thermal anomalies observed in 1983 by Nitsche and Straub [6] in a sounding rocket experiment to be eventually explained.

In this paper, we report direct evidence for this piston effect and study the velocity of the HBL. We indeed observed, both under weightlessness and under terrestrial conditions, a spectacular jetlike propagation from the HBL into the bulk fluid when heating is produced in a fissure or crevasse, as described below.

\section{FLUID VELOCITY AT THE EDGE OF A HOT BOUNDARY LAYER}

In the framework of a one-dimensional hydrodynamic model with a heating wall of surface area $S$ delivering a constant heat flux $\dot{Q}$, Zappoli and Carlès [7] established the velocity of the matter flow at the edge of a HBL:

$$
v_{0}=k \frac{\dot{Q}}{S},
$$

where

$$
k=\frac{\alpha_{P}}{\rho c_{P}}
$$

and

$$
\alpha_{P}=-\frac{1}{\rho}\left(\frac{\partial \rho}{\partial T}\right)_{P}
$$

is the isobaric thermal expansion coefficient; $c_{P}$ is the specific heat at constant pressure and $P$ is pressure. 


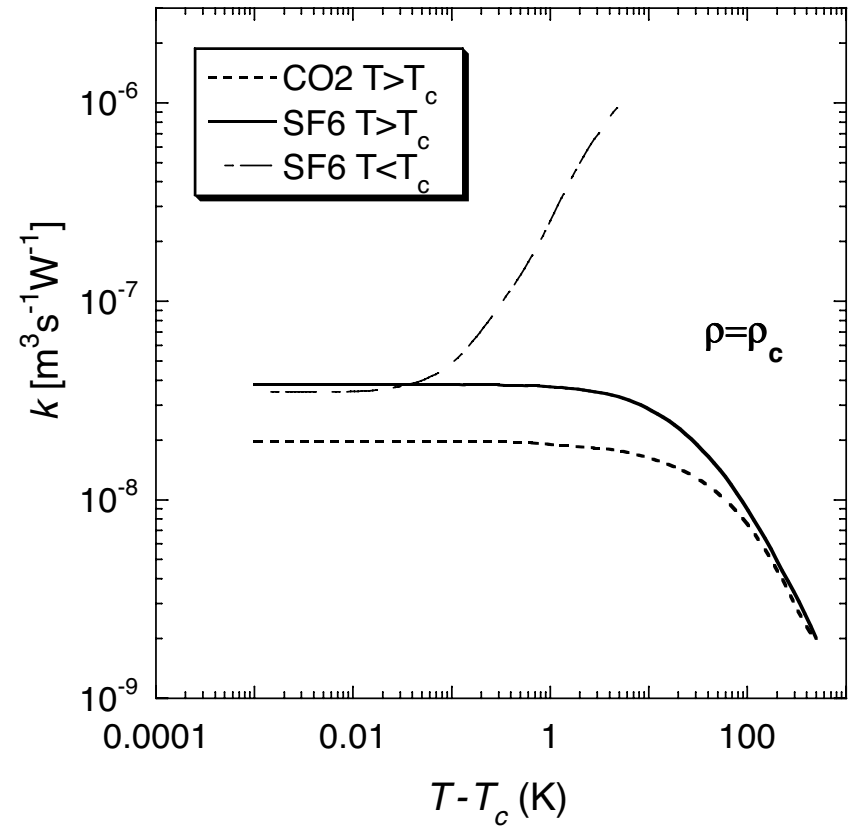

FIG. 1. The parameter $k=\alpha_{P} / \rho c_{P}$ is traced for $\mathrm{CO}_{2}$ and $\mathrm{SF}_{6}$ as a function of absolute temperature difference from $T_{c}$, along the critical isochore and for the vapor phase below $T_{c}$ (Log-log scales).

According to Eq. (1), $v_{0}$ is proportional to the injected heat flux per surface unit, $\dot{Q} / S$. This proportionality can explain the occurrence of the jet in a crevasse as observed in the following. The HBL velocity $v_{0}$ can indeed be locally very large when the heating surface is reduced at constant $\dot{Q}$. In addition, a crevasse geometry prevents the expansion of the fluid in the lateral directions and helps to generate a propagating fluid column.

In Fig. 1, the factor $k$ is traced for both $\mathrm{CO}_{2}$ and $\mathrm{SF}_{6}$ as a function of temperature along the critical isochore according to the corresponding equations of state $[8-10]$. This factor does not vary strongly near the critical point because the divergences of $c_{P}$ and $\alpha_{P}$ compensate each other. In fact, near $T_{c}$, Eq. (1) reduces to

$$
v_{0}=\frac{1}{T}\left(\frac{\partial T}{\partial P}\right) \frac{\dot{Q}}{S},
$$

in which $k \propto(\partial T / \partial P)_{\rho}$ is constant above $T_{c}$, as outlined by Garrabos et al. [11]. For $\mathrm{SF}_{6}, k$ is nearly two times higher than for $\mathrm{CO}_{2}$ (Fig. 1). Thus, for a given heat flux, larger velocity should be obtained in $\mathrm{SF}_{6}$. Below $T_{c}$, the $k$ parameter increases. However, a number of difficulties make the study of the two-phase domain extremely delicate. When heated, the liquid phase can indeed transform itself into vapor. In the vapor phase, another difficulty concerns the presence of thin wetting films on the thermistor and in the crevasse. We thus report on only a few data obtained at $T<T_{c}$ when, apparently, no wetting liquid phase was visible. The study then deals mainly with experiments carried out in the single-phase region.

\section{EXPERIMENTAL SETUP}

The experiments were performed in the automatic ALICE-1 facility [12], with the laboratory model on ground and the flight model onboard the MIR station.

The experimental cycles were carried out with two different fluids $\mathrm{CO}_{2}$ and $\mathrm{SF}_{6}$, both at critical density. Weightless and $1 g$ data were obtained with $\mathrm{SF}_{6}$ whereas only $1 g$ data were available for $\mathrm{CO}_{2}$.

The ALICE-1 instrument provides optical and thermal diagnostics. Here only the interferometry observation is of concern. Thermal control can be achieved within a few microkelvins in a temperature range $\left(20-70{ }^{\circ} \mathrm{C}\right.$ ) (for more details, see [12]). The experimental cells used for the $\mathrm{CO}_{2}$ and $\mathrm{SF}_{6}$ study are identical. They are made of a $\mathrm{CuCoBe}$ alloy. The internal fluid volume is a cylinder $(R=11.6 \mathrm{~mm}$ internal radius, $e=6.79 \mathrm{~mm}$ thickness) sandwiched between two parallel sapphire windows, the inner surface of one being coated with a dielectric mirror that forms one arm of a TwymannGreen interferometer. The $\mathrm{SF}_{6}$ cell is filled at critical density within $0.03 \%$ and its critical temperature $T_{c}=45.555{ }^{\circ} \mathrm{C}$ is detected before flight within $1 \mathrm{mK}$. Many previous experiments have shown that the $T_{c}$ drift with time was negligibly small in such samples. We thus impose the value in all our programmed experiments. Visual observations are indeed in agreement with the above value. The $\mathrm{CO}_{2}$ cell is filled at critical density within $0.5 \%$. Its critical temperature is $T_{c}$ $=31.051^{\circ} \mathrm{C}$. Note that the temperature sensors are not calibrated; the indicated temperature values are then only relative.

In each cell, temperature measurements inside the fluid sample are performed by two thermistors (Thermobeads, Serie B10, Thermometrics Inc., Edison, NJ, USA). The thermistors, labeled Th1 and Th2, respectively (see Fig. 3), have a time constant of about $10 \mathrm{~ms}$ and a maximum power rating of $10 \mathrm{~mW}$ in the temperature range considered here. Their electric resistance $R$ is a function of temperature with exponential behavior $R \sim \exp (1 / T)$ and nominal value $R=10 \mathrm{k} \Omega$ at $T=25^{\circ} \mathrm{C}$. Each thermobead consists of a miniature bead thermistor (made of metallic oxide semiconductors) that are hermetically sealed by means of specially selected glass coating. The miniature bead thermistor are connected to two opposite leads (made of platinum alloy) sintered inside the glass coating which forms a spheroidal pearl of $0.26 \mathrm{~mm}$ nominal diameter.

The thermistor Th2 can be monitored in a self-heating mode in order to induce a heat pulse of finite time duration $\Delta t$. The heat pulse is initiated by connecting the thermistor to a $U=10 \mathrm{~V}$ dc power supply of the ALICE facility. The dissipated power $P_{0}=U^{2} / R \approx \dot{Q}$ during the heat pulse varies with the initial temperature and $\Delta t$. The minimum initial heat power varies between $20 \mathrm{~mW}$ at $T \approx 30^{\circ} \mathrm{C}$ and $30 \mathrm{~mW}$ at $T \approx 45{ }^{\circ} \mathrm{C}$. In the present study, $\Delta t$ varies between $40 \mathrm{~ms}$ (minimum heating time) and $1.80 \mathrm{~s}$. In the latter case the heat pulse is made of 15 periods of $40 \mathrm{~ms}$ heating and $80 \mathrm{~ms}$ relaxation, to reduce the self-heating effect. It was noted that, when the heating thermistor is directly connected to the power supply of the ALICE facility, a divergence of the dissipated power is induced because the system is unstable: 

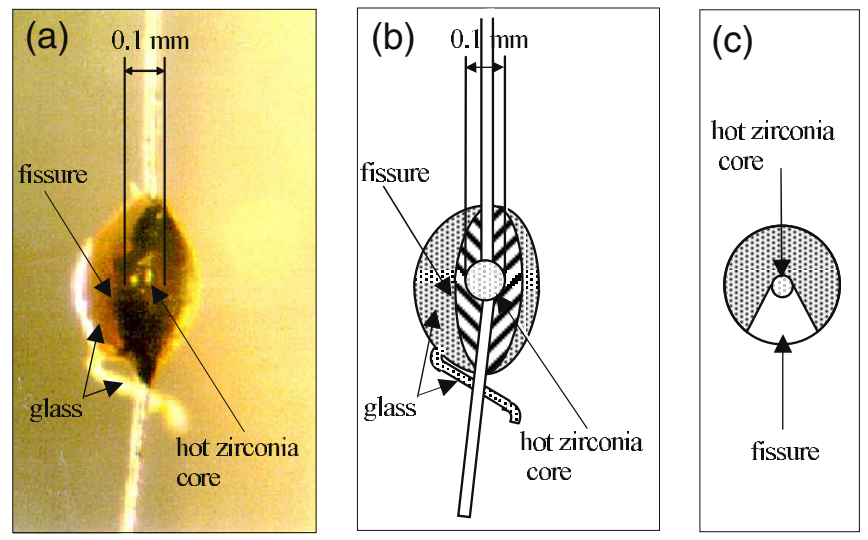

FIG. 2. (Color online) (a) Photo of the Th2 thermistor with the fissure and the central metal oxide sphere. (b) and (c) Sketch of the thermistor.

heating raises the thermistor temperature, and the resistivity decreases, which then results in a further increase of the dissipated power. This temperature rise can be important at the maximum power delivered by the ALICE facility. Values as large as $100{ }^{\circ} \mathrm{C}$ are currently measured after the heat pulse has stopped. According to the technical specifications, the thermistor can support without melting a quick temperature rise up to $600{ }^{\circ} \mathrm{C}$ in a "flash mode." However, the heating thermistors were locally damaged when heat pulses of maximum power were performed during the performance tests of the thermostat. As a result, a crack, resembling a crevasse, was created in the glass bead (Fig. 2). Around the edge of this crack, the surface changes its color from olivegreen to black. On the opposite face, a black stripe was visible at a location symmetrical to the fissure. The change of the surface color is a consequence of the high temperatures reached during heat pulses that burned parts of the thermistor surface.

It is exactly at the position of the open fissure that the jet flow appears, where the heating flux is concentrated on a small surface. From Fig. 2, the heating area $S_{\text {inner }}$ of the inner walls of the crack can be estimated to be $S_{\text {inner }} \leqslant 3$ $\times 10^{-8} \mathrm{~m}^{2}$

It was not possible to make such a detailed description of the similar crack in the thermistor of the flight sample $\left(\mathrm{SF}_{6}\right)$ due to its inappropriate position with respect to the direction of observation. However, a similar crack geometry was undoubtedly present as the jet occurred very repeatedly from the very same location.

On ground, the experiments concerning $\mathrm{CO}_{2}$ are carried out with a series resistance in order to reduce thermistor overheating. The ALICE power supply has been also replaced by an external stabilized source enabling pulses of different heating power $0 \leqslant P_{0} \leqslant 65 \mathrm{~mW}$ to be performed.

\section{OBSERVATIONS}

\section{A. Heat pulses of $\Delta t=40 \mathrm{~ms}$ under $1 \mathrm{~g}$ and microgravity conditions}

The jet starts systematically at the early beginning of the heating period. A propagating finger is already observed on

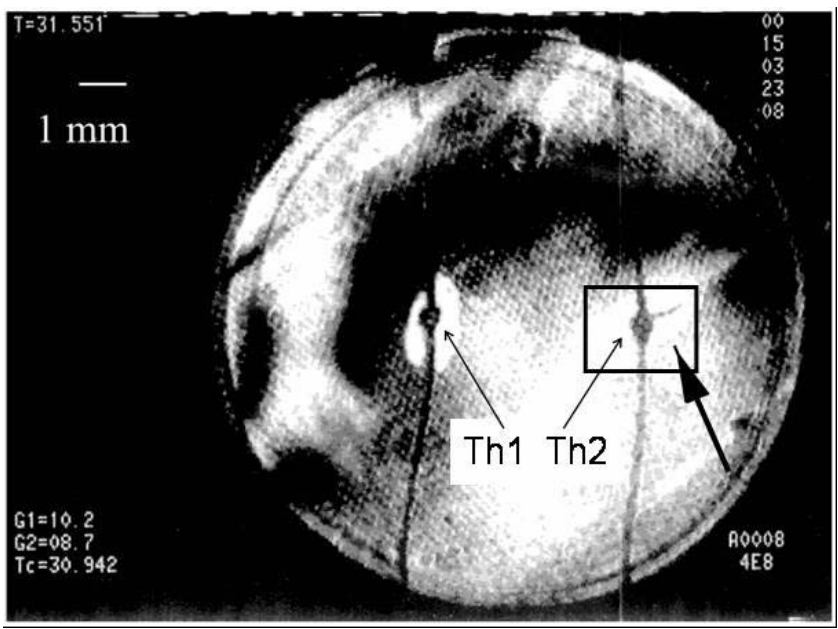

(a)

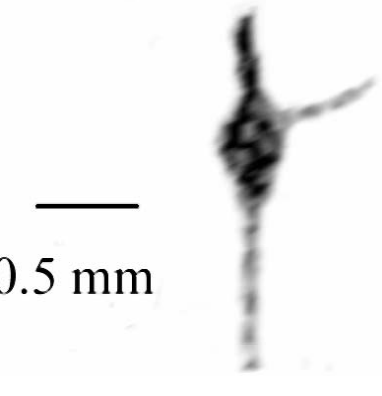

(b)

FIG. 3. Observation of a jet after a $40 \mathrm{~ms}$ heat pulse $\left(\mathrm{CO}_{2} ; 1 \mathrm{~g}\right.$ conditions; $T=T_{c}+500 \mathrm{mK}$ ). (a) The cell with two thermistors (Th1 and Th2). Th2 is also used as a heating source. The jet is indicated by a black arrow. The region shown by the black square is enlarged in (b). (The large black stripes are interference fringes. For clarity, regions around the thermistors and jet have been erased.)

the first video image within $40 \mathrm{~ms}$ after the start of the heat pulse. As there is no time correlation between the video picture and the start of the pulse, the exact time where the picture is taken can be any time after the start of the pulse, i.e., between 10 (thermistor time constant) and $50 \mathrm{~ms}$. The finger is observed both under $1 g$ (Fig. 3) and microgravity $(\mu g)$ (Fig. 4) conditions.

Under $1 g$ conditions, we have also studied the jet propagation with a $250 \mathrm{images} / \mathrm{s}$ high-speed camera. The jet propagation could be detected already within the first frame, i.e., $4 \mathrm{~ms}$ after the beginning of heating. The jet occurs at each heat pulse provided that a relaxation time between the heat pulses greater than 1 min (under $1 g$ conditions) is respected. The jet starts always at the same place and follows the same path.

Under $\mu g$ conditions, the evolution of a jet for a heat pulse at $T=T_{c}+2.15 \mathrm{~K}$ is shown in Figs. 4(a)-4(d). A fast propagation is detected within $40 \mathrm{~ms}$ after heating has started [Fig. 4(a)]. At this stage, only a very thin HBL can be observed at the thermistor surface, outside the crevasse. Further evolution is shown at $t=80 \mathrm{~ms}$ [Fig. 4(b)], where a "plume" has formed with lateral vortices due to viscous friction. (The vortices are hard to see on Fig. 4; one can detect their pres- 
(a)

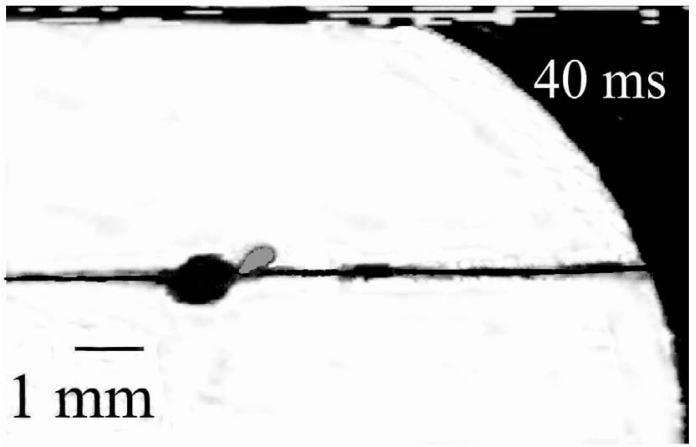

(b)

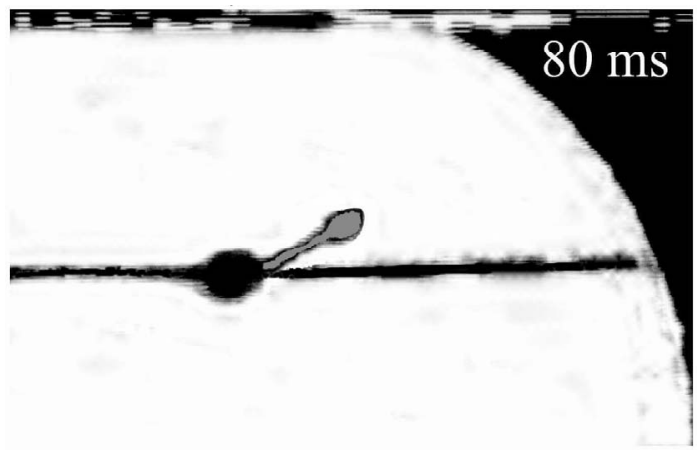

(c)

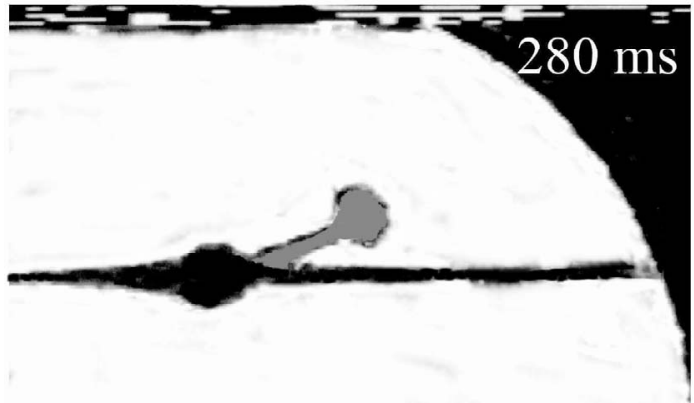

(d)

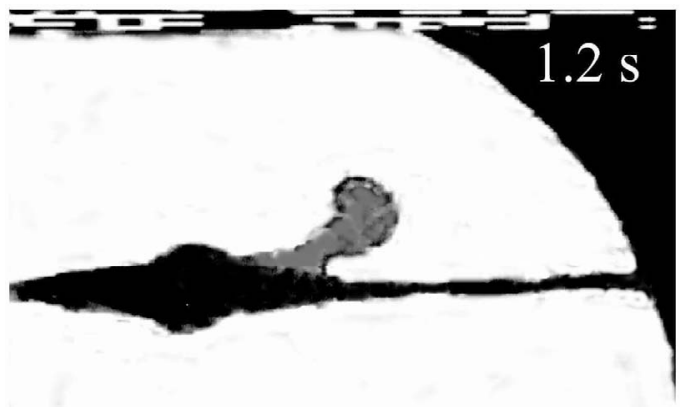

FIG. 4. The evolution of a jet observed in weightlessness after a $40 \mathrm{~ms}$ heat pulse $\left(\mathrm{SF}_{6} ; T=T_{c}+2.15 \mathrm{~K}\right)$, within $t=$ (a) 40, (b) 80, (c) 280, and (d) $1200 \mathrm{~ms}$ after the start of the pulse. (For clarity, regions around the thermistors and jet have been erased and the inside of the jet has been colored gray.)

ence by the mushroomlike shape of the head of the jet.) The jet propagation then slows down. A thick HBL is now visible around the thermistor and the threads at $t=280 \mathrm{~ms}$ [Fig. 4(c)]. The jet finally stops and starts to vanish by diffusion for $t \gtrsim 1 \mathrm{~s}$ [Fig. 4(d)].

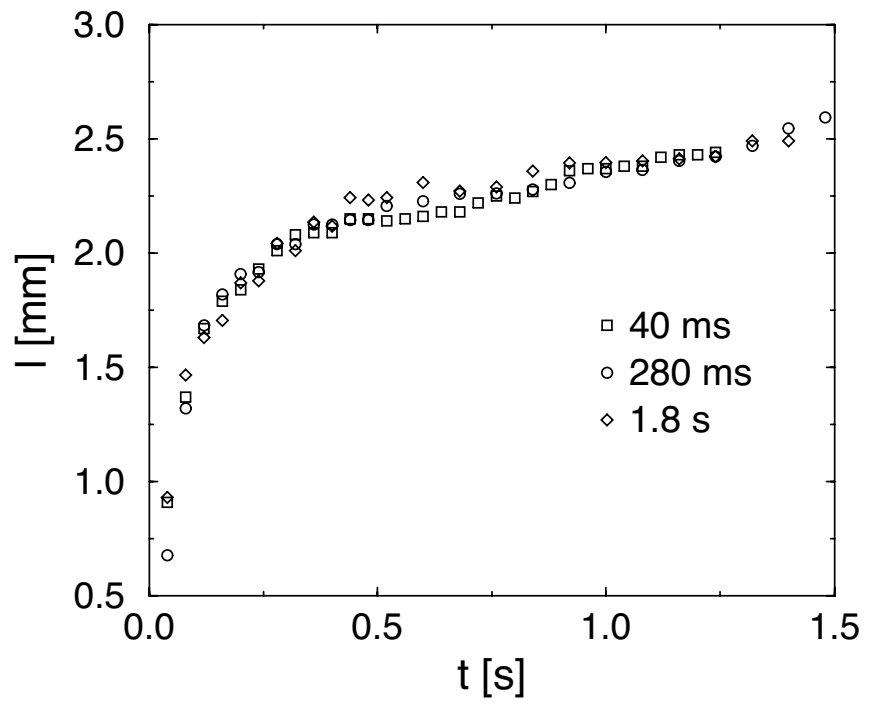

FIG. 5. Evolution of the jet length for three heat pulse durations $\left(\mathrm{SF}_{6} ; \mu g\right.$ conditions; $T=T_{c}+750 \mathrm{mK}$ ).

\section{B. Heat pulses of $\Delta t$ variable under $1 \mathrm{~g}$ and microgravity conditions}

The effect of heat pulse duration is shown in Fig. 5. The evolution of the jet length $l$ is plotted for three pulse durations 40 and $280 \mathrm{~ms}$ and $1.8 \mathrm{~s}$. The fluid is kept at the same initial temperature $T=T_{c}+750 \mathrm{mK}$. A striking result is that the heating time does not influence the jet behavior. Since a pulse of $40 \mathrm{~ms}$ duration already induces the same propagation than longer pulses, the phenomenon is then ruled by a characteristic time smaller than this $40 \mathrm{~ms}$ time (the minimum heating time in our setup).

From these experimental observations it is clear that the phenomenon is ruled by the initial thermal expansion of the fluid HBL inside the thermistor crevasse at the very beginning of the heat pulse. This is a process of a very short characteristic time and independent of the heating time as explained below. The fact that the heated fluid is confined in a cavitylike crevasse explains the jet formation and the higher initial fluid velocity (and subsequently longer propagation) when compared with the usual thin HBL that develops around a spherical heating source, as is the case in other places of the thermistor bead. The jet evolution does not depend on the heating time as a result of the limited fluid mass inside the cavity. Once the fluid is heated and expands, its temperature and density are no longer near the critical point and the fluid stops its expansion. What only matters is thus the initial velocity of the jet, which we investigate below.

\section{INITIAL VELOCITY}

The initial jet velocity $v_{0}$, as determined by the average value calculated within the first $40 \mathrm{~ms}$ after the start of the heat pulse, is plotted in Fig. 6 with respect to $T-T_{c}$. One can see that the evolution of the jet is only temperature dependent far from the critical point, in agreement with Eq. (1).

The values of $v_{0}$ for the $\mu g$ experiments are compared with measurements under $1 g$ conditions. Under terrestrial 


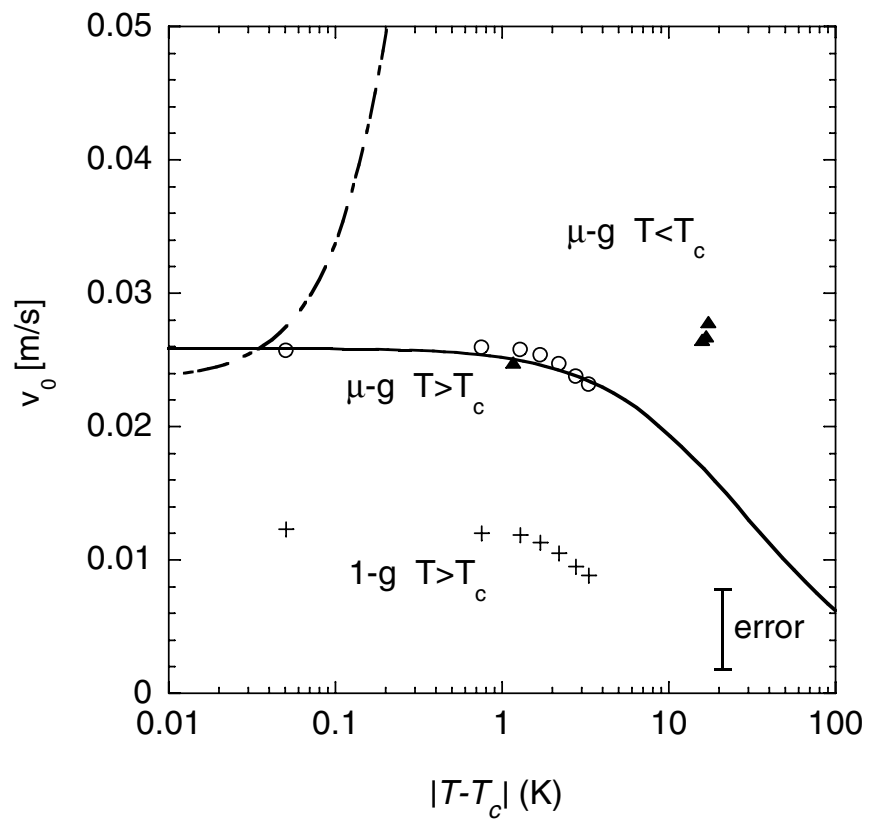

FIG. 6. Initial jet velocity $v_{0}$ as a function of temperature ( $T$ $>T_{c}, \mu g$, circles; $T>T_{c}, 1 g$, crosses; $T<T_{c}, \mu g$ triangles). The full $\left(T>T_{c}\right)$ and dashed $\left(T<T_{c}\right)$ lines are calculated from Eq. (1).

gravity, a strong convection induced by the heating thermistor makes the long-term behavior of the jet flow impossible to study. Accordingly, we concentrated our study only on the behavior of the initial velocity $v_{0}$. The $1 g$ and $\mu g$ data exhibited the same overall behavior; however, the $v_{0}$ values obtained on earth are approximately half those of the corresponding data obtained under weightlessness. Two possible explanations for the influence of the terrestrial gravity field can be found for this discrepancy.

First, although the average density of the cell is critical, density stratification under the earth gravitational field leads to an off-critical density at the thermistor location. In Fig. 3, the heating thermistor is, however, seen to be situated in the middle part of the sample cell, where density remains critical as $T_{c}$ is approached.

Second, convection (even not visible on the video at this stage) can disturb the expansion process of the fluid. A typical evolution time for convection can be given. It is approximately the time that a fluid element of size $r$ takes to move by buoyancy on the same length scale $r$. By using the wellknown Stokes formula, the convection velocity $V$ $=\rho r^{2} g \alpha_{P} \Delta T / \eta$. Here $\Delta T$ is the temperature rise and $\eta$ is the shear viscosity. At $1 \mathrm{~K}$ from $T_{c}$, for $\mathrm{CO}_{2}, \alpha_{P} \approx 1.4 \mathrm{~K}^{-1}$ and $\eta \approx 3.0 \times 10^{-5} \mathrm{Pas}$. The size $r$ is of the same order as the thermistor crevasse size, i.e., $\approx 0.1 \mathrm{~mm}$. The temperature rise $\Delta T$ is currently in the order of $50 \mathrm{~K}$ (the thermistor can even melt down and rupture, as shown by the presence of the crevasse), giving a time $\approx r / V \approx 1 \mu \mathrm{s}$. This time varies as $\alpha_{P}^{-1}$, i.e., it decreases strongly near $T_{c}$. It is therefore nearly impossible to get rid of convection on earth even on such a small length scale, a fact that explains the low $v_{0}$ value.

In Fig. 6 the data under $\mu g$, above and below $T_{c}$, are compared with the velocity of a HBL [Eq. (1)] with internal crevasse area $S \simeq 2.9 \times 10^{-8} \mathrm{~m}^{2}$ and heating power $\dot{Q}$

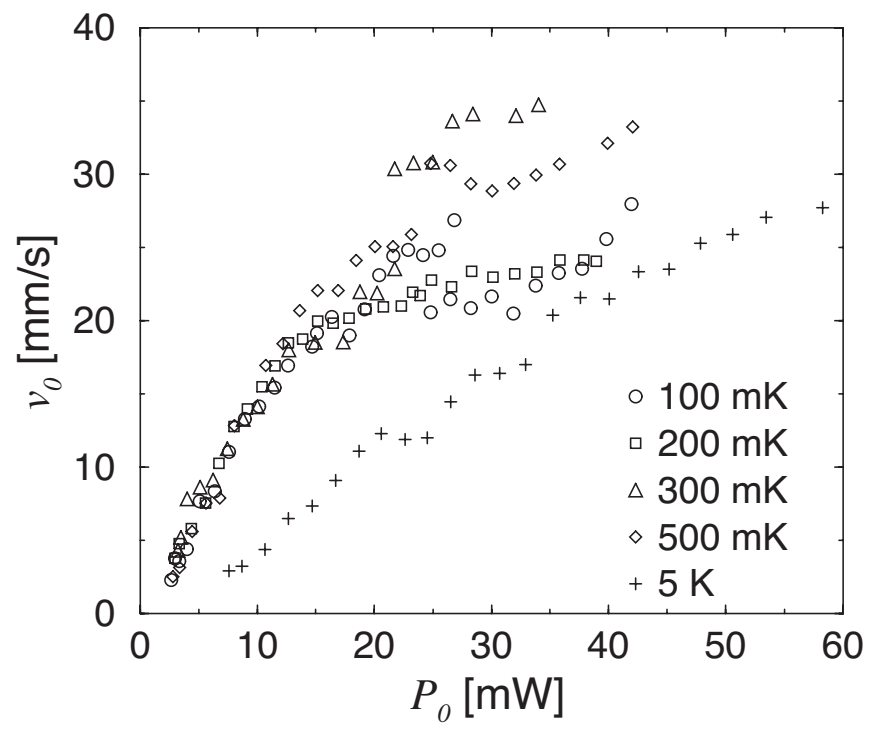

FIG. 7. Initial jet velocity $v_{0}$ at different $T-T_{c}$ as a function of the power rate $P_{0}\left(\mathrm{CO}_{2} ; 1 g\right)$.

$\simeq 20 \mathrm{~mW}$ (power density $\simeq 0.7 \mathrm{MW} \mathrm{m}^{-2}$ ). The agreement with the data at $T>T_{c}$ is excellent. However, the velocity measured in the vapor phase, below $T_{c}$, reveals an increase of $v_{0}$ with $\left|T-T_{c}\right|$. This is due to experimental difficulties in the two-phase region where phase separation and wetting phenomena complicate the thermal behavior.

Further information concerning $v_{0}$ are obtained with the $1 \mathrm{~g}$ measurements in the $\mathrm{CO}_{2}$ sample. Here the heating power is varied. In Fig. 7, the initial velocity $v_{0}$ is given for different temperatures as a function of the electric power $P_{0}$.

When $P_{0}$ is small, a linear relationship

$$
v_{0}=s P_{0},
$$

with $s$ a proportionality coefficient, is seen to hold. For high values of $P_{0}, v_{0}$ saturates, a fact that can be interpreted as the effect of increasing energy dissipation by heat diffusion from the "hot" jet fluid into the colder bulk fluid (see below the effect of diffusion).

The $s$ coefficient is seen to decrease for higher temperatures, i.e., the expansion of the jets becomes slower (and the propagation speed is slower). This behavior corresponds to what has been observed with $\mathrm{SF}_{6}$ in Fig. 6.

\section{JET DIAMETER}

The diameter $d$ of the jet is measured within $t=40 \mathrm{~ms}$ after the start of the heat pulse as a function of temperature in $\mathrm{CO}_{2}$ under terrestrial conditions (Fig. 8). It was found that, close to $T_{c}$, the diameter tends to have a value of $\approx 0.1 \mathrm{~mm}$. This value compares very well with the estimated size of the crevasse as detected on the thermistor (Fig. 2). When the temperature increases, $d$ grows according to a power law close to $\left(T-T_{c}\right)^{0.34}$, as demonstrated on the log-log plot in Fig. 8. The fact that $d$ grows faster far from $T_{c}$ confirms the process whereby a hot expanded fluid volume propagates (the jet) and from which heat diffuses radially into the colder 


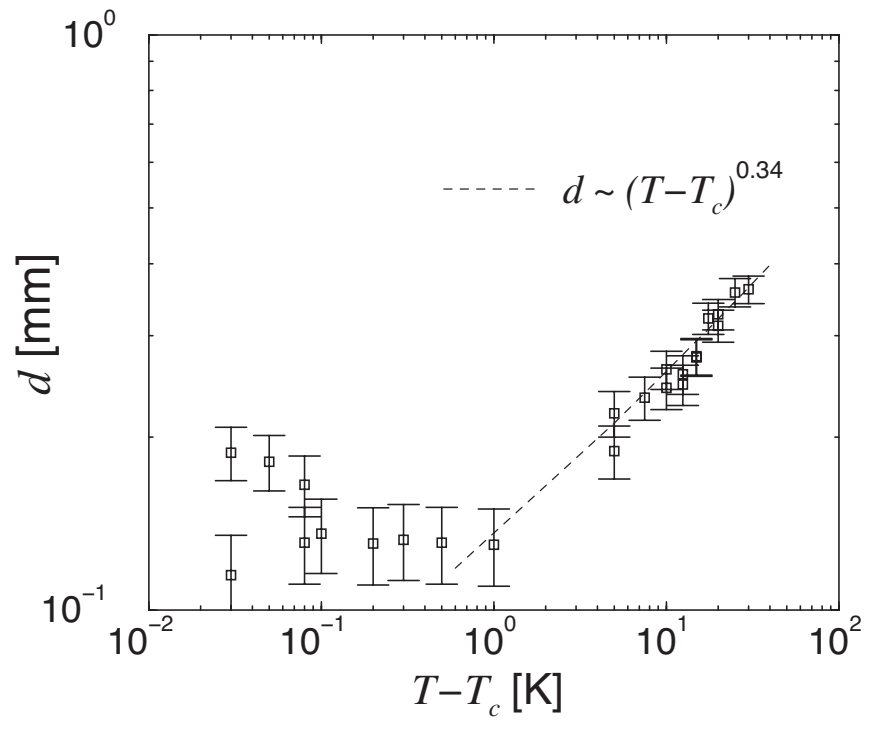

FIG. 8. Jet diameter $d$ within $40 \mathrm{~ms}$ after the start of a $40 \mathrm{~ms}$ pulse for different temperature differences from $T_{c}\left(\mathrm{CO}_{2} ; 1 \mathrm{~g}\right)$. The dashed line is the power law Eq. (5).

bulk fluid. Near $T_{c}$, where the thermal diffusivity $D_{T}$ vanishes, the jet diameter tends to the size of the thermistor defect. The thermal diffusivity scales with $T-T_{c}$ as

$$
D_{T} \propto\left(T-T_{c}\right)^{\gamma-\psi} \approx\left(T-T_{c}\right)^{0.67} .
$$

Here $\gamma=1.24$ is the critical exponent for $c_{P}$ and $\psi=0.57$ is the critical exponent for the thermal conductivity $[13,14]$.
The diameter $d$ should then obey the diffusion law

$$
d \propto \sqrt{D_{T}} \propto\left(T-T_{c}\right)^{(\gamma-\psi) / 2} \propto\left(T-T_{c}\right)^{0.34} .
$$

The data at $T-T_{c}>1 \mathrm{~K}$ are in agreement with such a power law with exponent 0.34 as reported in Fig. 8.

\section{CONCLUSIONS}

The key role of the expansion of the hot boundary layer (piston effect) in the heating process in near-critical, hypercompressible fluids can be spectacularly documented when a special geometry (here a crevasse in a heating thermistor) enables high density energy to be sent into the fluid. It results in a high velocity of the hot boundary layer expansion that looks like a "jet." The hydrodynamic nature of the piston effect manifests itself by the presence of this jet, whose behavior is seen to be in quantitative agreement with the hydrodynamic theory of the piston effect-provided, however, that the experiment were free of gravity effects. Such effects, by mixing the hydrodynamics of thermal convection and the piston effect, are indeed seen to reduce the expansion velocity.

We note that high fluid velocities can be triggered by only a very small amount of energy $\left(<10^{-7} \mathrm{~J}\right)$. Interesting applications concerning the management of fluids in space might follow from this study.

\section{ACKNOWLEDGMENTS}

We would like to thank M. Bonetti and V. Nikolayev for help. The studies were partly funded by the Centre National d'Etudes Spatiales.
[1] A. Onuki, H. Hao, and R. A. Ferrell, Phys. Rev. A 41, 2256 (1990); A. Onuki and R. A. Ferrell, Physica A 164, 245 (1990).

[2] P. Guenoun, B. Khalil, D. Beysens, Y. Garrabos, F. Kammoun, B. Le Neindre, and B. Zappoli, Phys. Rev. E 47, 1531 (1993).

[3] B. Zappoli, D. Bailly, Y. Garrabos, B. Le Neindre, P. Guenoun, and D. Beysens, Phys. Rev. A 41, 2264 (1990).

[4] R. P. Behringer, A. Onuki, and H. Meyer, J. Low Temp. Phys. 81, 71 (1990).

[5] H. Boukari, J. N. Shaumeyer, M. E. Briggs, and R. W. Gammon, Phys. Rev. A 41, 2260 (1990).

[6] K. Nitsche and J. Straub, Naturwiss. 73, 370 (1986).

[7] B. Zappoli and P. Carlès, Eur. J. Mech. B/Fluids 14, 41 (1995).

[8] J. V. Sengers, in Supercritical Fluids: Fundamentals for Appli- cation, edited by E. Kiran and J. M. H. Levelt Sengers (Kluwer, Dordrecht, 1994).

[9] G. X. Jin, Ph.D. thesis, University of Maryland, 1993 (unpublished).

[10] A. Abbaci and J. V. Sengers, University of Maryland, Technical Report No. BN 1111, 1990 (unpublished).

[11] Y. Garrabos, M. Bonetti, D. Beysens, F. Perrot, T. Fröhlich, P. Carlès, and B. Zappoli, Phys. Rev. E 57, 5665 (1998).

[12] J.-M. Laherrère and P. Koutsikides, Acta Astronaut. 29, 861 (1993).

[13] M. Bonetti, F. Perrot, D. Beysens, and Y. Garrabos, Phys. Rev. E 49, R4779 (1994).

[14] B. Le Neindre, R. Tufeu, P. Bury, and J. V. Sengers, Ber. Bunsenges. Phys. Chem. 77, 262 (1973). 